\title{
Relevance and Challenges of Traditional Birth Attendants' Practices in the Contemporary Midwifery Education and Practice: Evidence from Kwesimintsim District in Ghana
}

\author{
Hannah Yawson (PhD Student) \\ Principal, Lawra Nursing Training College
}

\begin{abstract}
The paper focused on exploring the relevance and challenges of Traditional Birth Attendants (TBAs) practice in the contemporary midwifery education and practice in Kwesimintsim District. This paper was conceived from the call to chase out TBAs from the maternal health care system. Two objectives emerged: one for the relevance and the other for the challenges. Quality analytical procedure was used with exploratory design. It emerged that despite the improved number of midwives, TBAs roles are still relevant in Ghana. It was also found that logistical inadequacies, difficulty in handling complications and strained relationship with midwives were the major challenges to the practice of the traditional birth attendants. It is recommended that TBAs should continually be married into midwifery services with long term piece meal withdrawal program. TBAs should also be resourced and partnership with Midwifery Training Colleges.
\end{abstract}

Keywords: Traditional Birth Attendants; Midwives, Pre-service Midwives; Relevance and Challenges.

DOI: $10.7176 / \mathrm{JHMN} / 61-08$

Publication date: April $30^{\text {th }} 2019$

\subsection{Introduction}

The prevention of child mortality continues to be on the radar of global health forum. As such the current Sustainable Development Goals, in its goal three (3) proposes to among other health related problems end child mortality by 2030 (Bryce, Black \& Victora, 2013). This agenda is very relevant due to the deteriorating infant mortality. According to WHO, it is estimated that each year around 2.7 million new-borns die in the first month of lives, worldwide and an estimated 303,000 women die from complications related to pregnancy or childbirth (Bayou, 2014). This number of death resulting from pregnancy and child birth is not only alarming but detrimental to upward scaling of global development.

In Ghana, Maternal Mortality Rate (MMR) is estimated at 510 per every 100,000 live birth. This figure far exceeds the SDG parameter. The frightening number of MMR and other pregnancy related complication calls for the need to build healthcare sector in a way that could rapidly respond to the challenges of child birth. One of the best strategies that has been identified to be crucial for saving the lives of pregnant women and as an indicator for progress in the reduction of maternal mortality is the utilisation of skilled birth care (Pfeiffer \& Mwaipopo, 2013; Ribeiro, Decio. 2014; Sarelin, 2014). This led to a shift in the midwifery system from traditional health structure to modernized health system where midwives or skilled birth attendants (SBAs) took over the responsibility of maternal health care delivery for the purpose of reducing mortality rates and provides standardised medical care to expectant mothers and postnatal services (Bayou, 2014). However, the SBAs have not been able to resolve the challenges and meet the expectations. Each year it is estimated that about four million newborns die in the first week of life, worldwide (World Health Organization, 2010). Additionally, according to Lawn, Cousens and Zupan (2005) an estimated 529,000 mothers die due to pregnancy-related causes.

These unfulfilled hopes called the world to rethink through their health policies. One of the options was to formally call the services of TBAs again. During the 1970s-1990s the WHO, UN and donor agencies mooted the idea of formally recognizing and training TBAs to complement efforts aimed at improving maternal and perinatal health in low and middle-income countries (Ana, 2011). However the integration was expected to be temporal as it was pending increasing in the number of SBAs (midwives). Since then, the roles of TBAs have been the subject of fierce debate among maternal health professionals worldwide and several repeated assessments have been conducted to ascertain whether the strategy works (Dzakpasu, Soremekun, Manu, Asbroek, Tawiah , Hurt , Fenty , Owusu-Agyei , 2012).

A lot of studies have belittled the impact of the introduction of partnering the TBAs. Sarelin, (2014) conducted a study on the subject and focused on the case of Malawi. The author revealed that the TBAs program failed to contribute to reducing mortality rate rather it worsened it. Malawi's Government has finally abandoned the program making the practice of traditional birth attendants (TBAs) illegal (Sarelin, 2014). The debate is still on going. Kelsey Harrison, a retired obstetrician and gynecologist argues on one side that TBAs do more harm than good partly because of their lack of education and the unhygienic environment in which they operate (Harrison, 2011).

Harrison's arguments are further grounded in a growing body of evidence that suggest that delivery in a health 
care institution or with skilled birth attendant (SBA) significantly reduces the chances of maternal and neonatal death compared to delivery by unskilled birth attendants ( Ana, 2011). With the current contemporary midwifery education and practice in Ghana where the number of midwives and health facilities have increased, should Ghana also abandon the program? What is the relevance of the TBAs practices currently?

Although many Ghanaian health professionals have provided assertions to call for the need to abandon the program, there are only a few empirical evidences to evaluate such calls and assertions. For instance, on the 15 th May, 2015, the Central Regional Health Directorate (CRHD) emphasized the danger associated with delivering at home and using the services of TBAs and urged expectant mothers to deliver at health facilities (Ghana News Agency, 2015). Some of the empirical evidences that support the abandoning of the TBAs program consistent with CRHD calls include Amjad et al, (2014) and Vyagusa et al (2013). These authors revealed that the instrument or materials used for helping in child delivery were either below standard or unhygienic. They also found that TBAs have low level of knowledge to assist the delivery of child birth which invariably has led to the failure to reduction infants mortality.

Contrary to the above, other empirical studies have shown that the practice of TBAs is still relevant (Ebuehi \& Akintujoye, 2012; Sarmento, 2014; Titaley et al, 2012). In fact, some researchers have revealed that TBAs play major role regarding maternal health care in in the most countries especially in the rural sides (Essendi, 2015; Kaingu, Oduma, \& Kanui, 2011; Nelms \& Gorski, 2006; Oshonwoh Ferdinand, Nwakwuo Geoffrey \& Ekiyor Christopher, 2014; Pfeiffer \& Mwaipopo, 2013; Selepe \& Thomas, 2000; Vyagusa, Mubyazi, \& Masatu, 2013). These TBAs belong to the informal healthcare system and are mostly unskilled and have limited tools (Shah, Brieger, \& Peters, 2011). Ebuehi and Akintujoye (2012) found that TBAs are cultural competent and some have good historic records which boost the confidence of the community in them. Besides, these empirical arguments, theoretically, assumptions from Health Believe Model (HBM), theory of culture care and Social Ecological Model (SEM) as reviewed in chapter two suggest that the practice of TBAs would die naturally if there enough health structures and professional attitude to render the practice irrelevant (Abbaszadeh, Borhani, \& Asadi, 2011; Ayele, Tesfa, Abebe, Tilahun \& Girma, 2012).

Following the conflicting assertions and empirical evidences coupled with strong theoretical support for the practice, it is important to undertake comprehensive assessment of the practice in the Ghanaian case. The assessment within the Ghanaian context is crucial as it has been observed from literature that Ghanaian women have traditionally preferred to deliver at home due to the fact that it is cheaper and easier as women who deliver at home mostly receive social support from their extended families and do not have to pay much for the delivery service (Crissman, Engmann, Adanu, Nimako, Crespo \& Moyer, 2013). According to reports from the Ghana Statistical Service (GSS), $62.9 \%$ of the total deliveries in the Northern Region occurred at home was assisted by nonskilled attendants, while in the Central and the Upper West Regions 39.1\% and 38.6\% births occurred at home were assisted by TBAs and relatives, respectively (Moyer, Adongo, Aborigo, Hodgson, Engmann \& DeVries, 2014). These statistics explained the significant roles TBAs play in the health of women and children in rural areas in Ghana. In spite of the forgoing statistics on the patronage of TBAs in Ghana, there exist several questions that need to be answered. For instance, with the contemporary midwifery education and practice where Ghana has produced and continuing to produce more qualified midwives, is the practice of TBAs still relevant? What are the challenges faced by expectant mothers and TBAs in the child delivery processes? Contributing in answering these questions could provide practical evidences about the extent of the relevance of TBAs, policy framework to support the integration and structure for abandonment. This what this paper seeks to address.

\subsection{Objectives}

i. Assess the relevance of TBA in contemporary midwifery in Kwesimintsim district

ii. Explore the challenges of traditional birth attendants practices in Kwesimintsim district

\subsection{Theoretical Literature}

The study is premised on the Health Belief Model (HBM). The Health Belief Model (HBM) is an intrapersonal within the individual, knowledge and beliefs theory used in health promotion to design intervention and prevention programs. It was designed in the 1950's and continues to be one of the most popular and widely used theories in intervention science (Green \& Murphy, 2014; Wright, Randall \& Grace Hayes, 2012). It provides guidelines for program development allowing planners to understand and address reasons for non-compliance. The HBM addresses four major components for compliance with recommended health action: perceived barriers of recommended health action, perceived benefits of recommended health action, perceived susceptibility of the disease, and perceived severity of the disease. In addition, there are modifying factors that can affect behaviour compliance. Some of the modifying factors include media, health professionals, personal relationships, incentives, and self-efficacy of recommended health action.

Social psychologists originally developed the Health Belief Model to predict the likelihood of a person taking recommended preventative health action and to understand a person's motivation and decision-making about 
seeking health services (Tones, Robinson \& Tilford, 2013). The Health Belief Model proposes that people will respond best to messages about health promotion or disease prevention when some conditions explicitly needed for change exist. HBM assumes that behaviour change occurs with the existence of numerous ideas at the same time. One of the ideas is that an individual recognises that there is enough reason to make a health concern relevant (perceived threat and severity). The subjective perception of the risk the individual is at from a state or condition (Gerend \& Shepherd, 2012).

A critical evaluation of the assumptions explain how community members would react to changes from accessing the services of the traditional birth attendants (presumed risky service) to skilled birth attendants (Presumed proper health service). From the theory it is evident that if a program for such change does not demonstrate the real reason to do so, then the communities would still marry the services of TBAs (Abbaszadeh, Borhani, \& Asadi, 2011; Ayele, Tesfa, Abebe, Tilahun \& Girma, 2012). Thus, despite the influx of midwives and the enhancement of contemporary midwifery education, it is possible that the TBAs services may be deemed relevance. Based on this theoretical analysis, it could be observed that the choice of TBAs by community women can overshadow that of trained nurses and midwives. Thus even in the event that TBAs are disbanded, the health belief orientation of certain women would make the use of TBAs still relevant. It is therefore important to fully understand and assess the relevance of TBAs in contemporary child delivery practices. This would obvious reveal areas within the practices of TBAs which needs attention and help in order to formulate polices and guidelines to streamline their operations.

Another construct that the HBM proposes is susceptibility and risk. Personal risk or susceptibility is one of the more powerful perceptions in prompting people to adopt healthier behaviour. It is assumed that the greater the perceived risk, the higher the likelihood of engaging in behaviour that would decrease the risk. This is what prompts men who have sex with men to be vaccinated against hepatitis B (Abbaszadeh, Borhani, \& Asadi, 2011) and to use condoms in an effort to decrease susceptibility to HIV infection (Taghdisi \& Nejadsadeghi, 2012). Perceived susceptibility motivates people to be vaccinated for influenza (Girma et al, 2012), to use sunscreen to prevent skin cancer, and to floss their teeth to prevent gum disease and tooth loss. It is only logical that when people believe they are at risk for a disease, they will be more likely to do something to prevent it from happening.

Relating this to the present paper, the target groups expected to change their health behaviour due to the perceived risk are the expectant mothers and the traditional birth attendants. The perceived risk may cause the expectant mothers to abandon the services of the TBAs for that of the midwives and TBAs willing to send complicated cases to the midwives. However, it is likely that these expectant mothers and TBAs would also evaluate their perceived risk for choosing the mainstream health centers. Some of these susceptibilities would be cost, convenience, trust, friendliness, recognitions and traditions.

This perceived risk associated with the use of TBAs could be one of the challenges that comes within the TBAs. Given proper guidelines and some level of training, the TBAs could run parallel with the orthodox birth attendants (Dako-Gyeke et al, 2013). This would not only reduce the pressure and inefficiencies in the hospital and health centres but also contribute to the reduction of maternal mortality. However, many people perceived the services of TBAs as risky and unhygienic (Girma et al, 2012; Taghdisi \& Nejadsadeghi, 2012). Where the perceived cost for the change is high, there is high degree of inconvenience, absence of trust from the new service providers (midwives), unfriendly atmosphere and embarrassment, they would evaluate these against the perceived risk and act appropriately. These suggest that evaluation of a good program has two tails and bi-direction. Thus, it is important to assess the relevance of this program from larger perspectives including TBAs and expectant mothers (Tavafian, Aghamolaei, Gregory \& Madani, 2011).

\subsection{Empirical Literature}

In addition to the forgoing theoretical underpinnings, there are some empirical studies that deals with the relevance and challenges of TBA in the contemporary practices. In 2012, Ebuehi and Akintujoye conducted a study to assess the role of traditional birth attendants (TBAs) in modern health care delivery in Edo State, Nigeria. The study sampled a total of 391 respondents comprising 48 TBAs, 309 childbearing mothers and 34 medical and paramedical professionals constituted the study sample. In-depth interviews and focus group discussions as well as validated questionnaires were the tools used for data collection.

The results revealed that respondents believe that TBAs can play meaningful roles in family planning, screening of high-risk pregnant mothers, fertility/infertility treatment and maternal and child care services. Rural dwellers prefer to use the services of TBAs, as compared to their urban counterparts. Reasons for the preference included TBAs, availability, accessibility, cheap services and rural dwellers' faith in the efficacy of their services. They recommended that there is, therefore, the need to restructure the training of TBAs as well as to fully integrate their services into the Nigerian orthodox healthcare delivery system especially as they affect rural settings.

The results of Ebuehi and Akintujoye (2012) present evidences for comparative analysis. Again the recommendation by Ebuehi and Akintujoye (2012) suggesting a full integration of TBAs practice into the Nigerian orthodox healthcare delivery system require further study. To effectively and successfully integrate the TBAs and 
the SBAs would require both the TBAs and SBAs to be corporative. The question is what are the challenges of these TBAs practice in the light of the existing SBAs?

In another study, Vyagusa, Mubyazi and Masatu (2013) conducted study to investigate the knowledge and practices of TBAs on EmoC in Kigoma Rural District, Tanzania and discusses policy implications on involving TBAs in maternal health services. The study employed a quantitative and qualitative technique for data collection and analysis depending on the nature of the information required where 157 TBAs were identified from several villages, interviewed and observed their level of knowledge and practice in health delivery. Summarising the findings of the Vyagusa et al (2013), it could be observed that majority of the TBAs have low level of knowledge on delivery complications and also signs of pregnancy complications. Moreover, it was observed that the instrument or materials used for helping in child delivery were either below standard or unhygienic. In general, it could be said that the activities of the TBAs from Tanzania, as indicated by this study does not contribute positively to the reduction of infant mortality and antenatal and post-natal crisis.

It is evident in the study that major challenges in TBAs practices are knowledge and logistics. Since majority of the TBAs are aged and have no formal education, the normal trend of academic training would still not yield the needed results. Better still some of the TBAs might not be interested in the content and mode of the existing training. All these predictions can be confirmed only through empirical analysis. It is against this that this study seeks to explore the training needs of the TBAs from their own perspectives and the mode of preferred training. This would make any program on these TBAs quite effective. Furthermore Vyagusa et al (2013) identified logics as challenge to TBAs practice. Could it be same in Ghana or other critical challenges exist? The present study seeks to provide the answer

In Malawi, a descriptive study was conducted in which 12 women were interviewed regarding their perceptions of perinatal care by Kumbani, Bjune, Chirwa, Malata, and Odland (2013). All 12 women had delivered at home and stated that they had wanted to give birth in a health facility. However, the women observed that health care accessibility was a main problem in rural areas (Kumbani et al., 2013).

In the emerging economy of Timor-Leste, Sarmento (2014), conducted a similar study and observed that traditional birth attendants have performed wide variety of tasks including outreach and case finding, health and patient education, referrals, home visits and care management. Evidence indicated that there were, to varying degrees, positive associations between traditional birth attendance training and maternity care. Traditional birth attendance training was found to be associated with significant increases in attributes such as knowledge, attitude, behavior, advice for antenatal care, and pregnancy outcomes. However, some challenges faced by traditional birth attendants' role in encouraging women to go to health center for preventive services would be the compliance and refusal of the referral. The implementation case study from Timor-Leste shows that integrating traditional birth attendance into a national healthcare system through Family Health Promoter program has been problematic.

Also, Asweto, Aluoch, Obonyo, and Ouma (2014) conducted a study in Kenya and found that that women in rural areas paid fewer visits to the health facility, citing distance as a barrier. The authors further suggested that physical remoteness and scarcity of health facilities and infrastructure are obstacles to seeking maternal health care among rural women. The implication of the study by Asweto et al is that ordinarily, women within the rural areas would have patronized the health facilities for maternal care if accessibility was easier and cost cheaper. In the case of the Kwesimintim district, could the services of TBAs be used in remote areas to supplement the effort of the skilled birth attendants.

In the Ghanaian context, the studies on TBAs are still in the infancy. Ganle (2015) conducted a study on the implications for Ghanaian maternal and newborn health for chasing out TBAs. The study revealed that midwives criticize the practice of TBAs and do not support marrying the TBAs into the modern health system. Ganle (2015), however revealed that given the low number of skilled birth attendants (SBAs), it is neither achievable nor feasible to ensure that all expectant mothers are attended to by SBAs. Ganle (2015)' study is one of the few studies which has boldly sought to evaluate the TBAs practice. However, are the relevance of TBAs limited to the number of SBAs? A critical review of the HBM theory suggests that cultural and societal norms could heighten the relevance of TBAs even when SBAs number improves. This paper seeks to explore some of these factors.

Adatara, Afaya,Baku, Salia, and Asempah (2018) also conducted a study on the experiences and the roles of TBAs in maternal health care in some rural areas of the northern part of Ghana. It was found that the main roles of the TBAs are conducting deliveries at home, providing health education to the expectant and lactating mothers on nutrition, arranging for means of transportation and accompanying the expectant mothers in labour to their health facilities, providing counseling and psychological support to expectant and lactating mothers. The authors concluded that TBAs roles are critical in maternity in rural and remote areas in Ghana. It was recommended that SBAs should collaborate with TBAs in the rural and deprived communities in providing quality and culturally accepted care in these areas.

The literature review has revealed theoretical and empirical areas within which the focus of the study falls. The HBM has underscore the importance of TBA from the cultural perspective and stressed the need to streamline its operations to conform with government policies and procedures. Moreover, it explained the challenges with 
regards to the possible risk that TBAs pose through their inability to handle complicated pregnancy cases. The empirical studies have revealed some the relevance and challenges that exist in some jurisdictions.

\subsection{Design and Methods}

Following the existing literature on the subject of investigation, this paper describes relevant methods and techniques in conducting the study. The key methodological issues considered in this paper are discussed as follows:

\subsection{Research Design}

A qualitative explorative design was used to examine relevance and challenges of contemporary traditional birth attendants practice in Kwesimintsim district. Exploratory design involves requires the search for individuals with adequate knowledge about the subject under investigation, solicit their views, opinions, beliefs and perceptions of the said subject. It also assists the researcher to formulate and understand the problems and clarify concepts.

\subsection{Study Population and Sampling Methods}

The target population of the study is the Traditional Birth Attendants (TBAs), expectant mothers, midwives, preservice midwives and the administrators from the Kwesimintsim in western region. The target groups are those who have adequate knowledge about the subject. The total population of the target is not available. However, it is estimated that the total TBAs in the whole Sekondi-Tarkoradi Metropolis for which Kwesimintsim district is a part is 186 (Ghanalocalassemblies.com). The study used 12 TBAs in the four communities, 24 expectant mothers, 10 pre-service midwives, the midwives of the health facilities available estimated at 8 and health administrators estimated as 6 . In total the sample size of 60 is used for the study. Having determined the sampling size, the study proceeds to use purposive techniques to select the participants.

\subsection{Data Collection Procedure}

Data were collected through semi-structured individual interviews using a guide. The interview guide contained questions that inquired about the relevance and challenges of the role of TBAs in maternal health care in the areas. Semi-structured interviews were also appropriate for this study because the researchers required detailed insights from participants. The in-depth interviews allowed the researchers the opportunity to define the areas to be explored and to probe further to pursue an idea or response in detail. The interviews were done face-to-face with participants at their various homes and at their convenient time which lasted between 30 to 45 minutes. The interviews were recorded with the permission of participants.

\subsection{Data analysis}

The thematic analysis approach was used for the analysis the data. The study examined the qualitative data, which were then categorized based on prominent theme patterns expressed in the text of the individual interviews (Kumbani et al., 2013). The study broke down the narrative data into smaller units, coding, and later naming the units according to the content to which they represent and coded material based on shared concepts and their meanings. All transcripts were read and analysed in a three-stage process of data analysis and synthesis (De Vos, Strydom, Fouche, 2009). In the first stage, the study used the exploratory method of holistic coding for the first coding cycle. After reading all the transcripts. The verbatim transcripts of the individual interviews were coded into different units. In the second stage of data analysis and synthesis, the focused coding method was used for the second coding cycle. Finally, based on the coding the primary researcher identified themes that integrated substantial sets of the coding units. Sets of categories were created to describe the predominant themes that emerged from the text (Creswell, 2009).

\subsection{Results and Discussions}

This section of the paper is presented in two folds. The first part reports the results from the qualitative analysis. This is done through the thematic procedures. The second part applies the results to the specific objectives of this paper and discusses them. Following the thematic analysis coupled with the research objectives, two broad themes and five (5) sub-themes emerged from the data analysis. The broad themes are: Relevance of TBAs practices, b) challenges in TBA practices. The five sub-themes are:
i. $\quad$ Choice to attend to TBAs
ii. Benefits of TBAs practices
iii. Logistical inadequacies
iv. Complications in TBAs practices
v. Constraints in TBAs practices 


\subsection{Theme one: Relevance of Traditional Birth Attendants Practice}

This theme describes the relevance of the practice of traditional birth attendants (TBAs) in child delivery. The participants revealed the relevance of the TBAs practices through the in-depth interviews. Each of the interviewed TBAs participants had the desire to succeed in every service they provide to expectant mothers and they were willing to put forth extra time and effort to make it happen. This theme has two sub themes which included: choice to attend and benefits of TBAs services.

\subsubsection{Sub-theme 1: Choice to Attend}

Participants revealed that their choices for attending the services of Traditional Birth Attendants include experiences and historical records, cost and convenience. According to some of the TBAs interviewed revealed that because they are well known and their skills and abilities are not hidden expectants mothers within their areas would not go through the pregnancy process without their advice. Even those mothers who use the hospitals as the primary health care still come to us for assistance. One of the TBAs stated:

"Mothers would say please assist us with herbs to quicken the delivery... "(TBA \# 3)

"Sometimes I am surprise myself, even some highly educated persons with means would travel with their wives only to see me for examination and advice because of my experience and the successes I have achieved over the number of years in practice" TBA \# 5)

The study also revealed that some mothers were not comfortable to receive health service from people they barely know. They choose the TBAs because they are part of their communities and therefore know their histories, abilities and skills. One expectant mother explained:

"The TBA that I have been receiving services from has helped almost every woman in my family to deliver successfully without any problem, so how could I choose any other person" (EM\# 4)

"I once wanted to deliver at the centre here but wanted to be sure whether the young lady (community midwife) can really help me deliver successfully. So I visited and wanted to know how many years she has been practicing and the number of successful deliveries he has had. In fact I didn't like how this young lady treated my questions so I have to stick to the traditional woman (TBA) in this community" (EM\# 9)

Another basis for choosing the services of TBAs found in the study was the cost associated with using traditional birth attendants' services versus the primary health care centre. Majority of the TBAs, EM (expectant mothers), midwives (MW) and pre-service midwives (PSMW) expressed cost of delivery as one of the major bases that cause expectant mothers to call for the services of TBAs in their communities.

"People like my service because I don't charge huge. Not until recently, I was not charging any fee for the services I render. The people had to give me something out from their own heart and whatever they give I accept without any question........Since I started charging some little fees, the number of deliveries have reduced" (TBA \# 1)

Some mothers also revealed their ordeal in relation to cost of delivery at hospital or clinics.

"I delivered my third child at a clinic, though I had health insurance my husband paid GHC65.00 excluding the taxi I chartered in and out of the clinic, we could have saved some of these monies if we had delivered at home" (EM \# 6)

"I'm really struggling to meet my daily expenses at home, how can you expect someone like me to pay a midwife? That place (health centre) is for those who have extra income to spend" (EM \# 11)

"I wanted to deliver my child at a hospital but I was given a tall list of items to be brought to the hospital when I come to deliver. I could not afford to buy all those items so I stopped going to that hospital' (EM \# 13)

Even some of the midwives and pre-service midwives (i.e. students) stated that cost of medical services could be one of the reasons why still some expectant mothers continue to use the TBAs services.

"Although I have not directly dealt with expectant mothers, in some of my practicals, I learnt that some of the mothers find it extremely difficult to meet the hospital cost. Some of the mothers had to overstay because relatives couldn't bail them out-----" (PSMW \# 2)

"Some of the women start their antenatal with us but when we provide them with the list of items to bring during their delivery, they stop coming. Our delivery rates are low" (MW \# 1)

The third key base for choosing the services of TBAs is the convenience in using the service. The results of the study indicated that distance to health centre and time constraints detest the usage of the health centres. It was 
evident in the interviews that most of the expectant mothers perceive the TBAs as more convenient as they can be called upon anytime to visit their homes when their services are needed. Since the TBAs are closer the expectant mothers do not need to travel in their condition to health facilities.

"The health service centre where I can receive assistants from the midwife is too far away......... The midwives are too bossy to contact them at ten o'clock at night. It is quite impossible for them to listen to you............Better to call for the service of a traditional birth attendant" (EM \# 17)

"I believe you can see our road network.......... It becomes worst during the raining season. If you were in my shoes or that of my husband will you risk your life through these challenges and drive through to the health centre for midwife you barely know when an experienced traditional birth attendant is just around your home?"(EM \# 21)

"Sometimes you will be in bed and hear your name, quickly you will have to wake up and attend to the person. No matter how deep your sleep may be. It is a custom and practice and the gods will not give you peace if you relent. Even if the woman has not been receiving assistance from you, still you have to wake up to help" (TBA \# 1)

Despite most of the expectant mothers claimed they prefer the TBAs, other mothers especially those in relatively urban centred as well as those closer to health facilities continue to use the services of the midwives in the health centres. One woman explained.

"................ I am told about the danger associated with home delivery so I can't risk my life and that of my baby.......... My husband and I would do everything not to missed my antenatal period with my midwife” (EM \# 1)

"What will happen when you meet complications. In fact neither I nor will I advise anybody to rely on the TBAs the cost and location cannot be used as excuse" (EM \# 3)

"How could you possibly know the position of your baby without the midwife? How do you know and manage your HB if you don't use the health facilities? using the services of a midwife or health facility is priceless? $(\mathrm{EM} \#$ 2)

Some also chooses to deliver at health facilities due to relationship with professionals of the facilities and proximity to the homes. Some of these women explained:

"................. at best I could only use the herbs from these women (TBAs).......they are good in this, However, I can't rely on them to deliver my baby. ............. I really know some of the midwives are rude but I have not encountered them personally possibly because I have a friend in the facility I used"' (EM \# 15)

"................I was using the services of a TBA until I moved in here. As you can see this is just the health centre and the madam (midwife) has been a family friend and understands our situation so currently I use the health centre. I delivered my third child here and will deliver this here too" (EM \# 23)

\subsubsection{Sub-theme 2: Benefits of Traditional Birth Attendants}

TBAs play pivotal roles in health delivery. The study showed that the TBAs have the ability to identify early pregnancy, pregnancy-related complications, counselling, referrals and provide herbs to assist expectant mothers. Many TBAs have rich experience and sound wisdom to provide extra ordinary services.

"When I conceived my first child I was going about on my normal duties without knowing I was pregnant. .............. One morning on my way to the market I saw our family friend who is TBA, she called me and told me I should be careful not to hurt the baby. I didn't get her right. So I paid her visit on my way home when she spoke with me in plain language. I could have got miscarriage if I didn't meet her" (EM \#10)

"Some women over rely on menstrual periods as evidence of pregnancy. However this is not always the case. My experiences I learnt from my grandmother have given me other key visible signs of pregnancy. ................ tell people they are pregnant when even they still experience their usual menses. .........It sometimes saddens me when I see pregnant women risking the lives of their babies because of ignorant------” (TBA \#8)

Some of the skilled birth attendants (SBAs) admitted that they have witnessed a scene where a TBA has just observed a pregnant woman and predicted correctly the kind of complication likely to follow the woman.

"When I was a kid, my mother used to diagnose some expectant mothers, identify a complication and specifically ask the mother to run some checks at hospital. Some of these women come back and confirm such complications (MW \#3) 
"My grandmother sometimes stops by a pregnant, takes the meal she is eating and discard.......She sometimes exclaims, why are you bringing problem upon yourself----I didn't understand her actions before but now I do through my career"(PSMW \#7)

The study found that despite the tension which sometimes occurs between the TBAs and the SBAs, some of the TBAs see as their religious duty to make quick referrals of mothers to nearest health centres. Thus, the TBAs play crucial co-ordination roles despite strained relationship with some of the SBAs.

"The gods give the knowledge to go by my normal duties. ........ what I cannot do the spirit will tell me and immediately refer the mother to the nearest clinic for safe delivery (TBA \#1)

"I do like my TBA because she gives me the best advice. My husband and I never trusted the SBAs, however, now we use the two services concurrently (EM \#14)

The results from the first theme and sub-themes relate to the first specific objective of this paper. The objective sought to assess the relevance of TBA in contemporary midwifery practice. This objective was important given the high number of enrolment in midwifery education annually and the continuous practice. It was evident from the results that most of the participants including the expectant mothers, TBAs and health centre administrators viewed the practice of TBAs as quite relevants. Thus, inspite of the availability of midwives in health centres, the TBAs practices were still seen as relevant.

Some of the contributing factors included cost, historic records, experiences, confidence and convenience. The communities believed that they are very well aware of the achievements of the TBAs and also know their background. Therefore they had strong confidence in them and would continue to choose them over midwives they barely know. Furthermore some of the trained TBAs co-ordinate with some midwives and advise their patients to visit the health centres. It was revealed that some expectant mothers visit health centres because of the recommendadtion from their trusted TBAs. Phyical accessibility to health centres was also a factor. The participants revealed that it is risky and costly to travel on long distance on roads which are immotorable especially during raining season. Other reasons included mistreatment the expectant mothers receive at the various health centres and traditions. TBAs shared the traditions of the community and prepare to protect the traditions, therefore they could confidently avail themselves for their services.

These findings have theoretical significance. The findings are consistent with the theoretical assumption of the Health Belief Model (HBM). HBM assumes that behaviour change occurs when there are enough reasons to do so (Tones, Robinson \& Tilford, 2013). According to the proponents, if the reasons are not favourable, it would render the health concern irrelevant (Gerend \& Shepherd, 2012). Therefore, the monetary cost of the change, cost of abandoning traditions, physical access and ill-treatments associated with delivery outside home make the change irrelevant. Thus, high rate of endorsing the TBAs given these conditions provide empirical evidences to support HBM.

Besides the theoretical contributions, the results confirm the findings and conclusions drawn by Titaley, Dibley and Roberts (2012). The study conducted by Titaley et al (2012) revealed that the use of traditional birth attendants and home delivery were preferable for community members despite the availability of the village midwife in the village. The authors also identified that physical distance and financial limitations were two major constraints that make the practice of TBAs still relevance. The findings in this study also affirmed the results of Ebuehi and Akintujoye (2012).

It is therefore concluded that despite the deepening in the contemporary education of the midwives and coverage of their practice, the practice of traditional birth attendants are still relevant to the community members. The implications of the findings are that health administrators and policy makers should strengthening the partnership programme and co-ordination between the TBAs and the health care system. Since the attitudes of the SBAs (midwives and pre-service midwives) have contributed to the relevance of the TBAs, the policy makers and administrators should revive a workable attitudinal changes right from their training in colleges to practice.

\subsection{Theme two: Challenges in TBAs practices}

This theme summarises the contents of the responses from the interviews which demonstrated the challenges the traditional birth attendants face in delivering their services to the mothers. The practical challenge as a theme was developed from three sub-themes. These sub-themes include logical inadequacies, complications and general constraints.

\subsubsection{Sub-theme 1: Logistical Inadequacy}

The TBAs believed that although they were following traditions and practices to maintain the family professions, their services are serving the community and require logical support. Some of the TBAs indicated that though they 
had gone through training by the government (ministry of health), they did not receive the needed logistics for their works.

"I sometimes use plastic bags to cover my hands during child deliveries since I do not have gloves..... "(TBA \#4)

"There have been some occasions when I had to use my bare hands to support child delivery..... I know it is not right but at times that is the best option" (TBA \#6)

During our training, they told us to sterilise our equipment, however, I don't have the necessary equipment to do so. ......... I do clean them in my own way and reuse though the government is saying it is not ideal" (TBA \#2) “ (TBA \#7)

\subsubsection{Sub-theme 2: Complications in Delivery}

The findings revealed that one of the major challenges of TBAs practice is the complications they identify on expectant mothers. According to most of the trained TBAs, their training focused on identifying complicated and emergency cases and make quick referrals. However, some of them indicated that they often received abusive words from the midwives when they send complicated cases to them. Some of these midwives deliberately delays in attending to their patients because they didn't come straight away to the health centre.

"I had to develop personal relationship with one midwife who I refer my cases to..........Initially like other midwives she was finding it difficult accepting why I had to try delivery at home. Now she has been accepting my referrals" (TBA \#4)

"I had some difficulty in delivery at one night around 11pm and was rushed to the health centre here with my husband and the TBA I have been seeing. I nearly died because the midwife was annoyed with us. She wanted us to have come directly to see her without trying the home delivery............ but I am used to the safe home delivery. My husband had to beg the young lady" (EM \#10)

\subsubsection{Sub-theme 3: General Constraints to TBAs Practice}

It was revealed that another major challenge of TBAs practice was constraints to clear scope of practice. Some of the trained TBAs explained that they were directed on their responsibilities in the health care system. However, since there are no clear policies for their practices some of the health care centres see their practices as illegal. They sometimes receive threats from the midwives. This does not motivate them to attend any new training sessions. Again, they also explained that because there is no clear policy and scope of operation, the extent of practice is dependent on the convictions of the individual TBA which some thought was not what they were taught.

"I sent an expectant mother to the health centre myself and this young lady (midwife) threatened to call the police to arrest me should I continue to practice home delivery......... work that our gods approve” (TBA\#3)

"The old women (TBAs) in the communities make our work very difficult. They keep the expectant mothers in the house and bring them only in emergency cases difficult to handle sometimes. .......... have cautioned some of these mothers that if they rely on these old women I would not attend to them, though I can't really do it but threatening them may be helpful" (MW\#1)

Some trained and certified TBAs said they have a lot of concerns to raise but do not know the appropriate quarters to send their grievances. They displayed their displeasure about how some midwives they perceive as their granddaughters could treat them with contempt.

The findings in respect of the second theme and the associated sub-themes relate to the second specific objective of this paper. The second objective of this study focused on exploring the challenges of traditional birth attendants' practices. This objective was relevant because early detection of challenges and provision of appropriate recommendations may minimise the potential threats to the masses as there is still a high level of attachment of community members to the TBAs practice.

The study found that the main challenges associated with TBA prcatice were logistical inadequacy, difficulty ith handling complications and challenges of acceptance by the SBAs. It was revealed that majority of the TBAs including the trained TBAs do not have the requisite logistics for safe practices. The level of hygienic practice was questionable. It was surprising to find that some even deliver with their bare hands and plastic bags. Although the trained TBAs were ready to sterilise their equipment as they were taught but logistics to do so were not available and they do not have the means to provide these logistics themselves.

Another challenges that the TBAs encounter in their practice is how to deal with complications they identify 
on expectant mothers. The TBAs indicated that although they have been told to refer complications to the health centres, their efforts are often strained by awkward and ill-treatment from the midwives in the health facilities. Although there is formal partnership programme, the findings suggested that most of these midwives and preservice midwives are not aware. It was also evident that some of these TBAs find it difficult to compromise on their traditions.

The results were consistent with the results of Yidana and Issahaku (2014). Yidana and Issahaku (2014) also concluded that one of the major challenges of integrating the traditional practice into the main stream health care is how to cohesion between TBAs and the SBAs. Similarly, the results were in line with the challenges identified in the study conducted by Ebuehi, and Akintujoye (2012) in Nigeria where it was revealed that the challenge on relying on TBAs practice is complication risk and how to respond to the risk.

Furthermore the findings revealed by Siaw and Siaw (2013) agreed with what were found in the present study. These authors also identified challenges in referrals as one of the major difficult in institutionalising the TBAs practice. It is therefore concluded from this objective that despite the relevance and the extent of marriage attachment by community members to the TBAs, the program has some major challenges such as logistical, complications and strains in referrals or relationship with main stream health care system. The implication is that the health administrators have more works to do if the plan to partner the traditional practice is to be sustained. The findings also imply that the level of awareness and communication between the partners are very low as evidenced in the low level of acceptance by midwives and pre-service midwives.

\subsection{Summary and Conclusions}

The main purpose of this paper was to assess the relevance and challenges of traditional birth attendants practice in the contemporary midwifery education and practice in Kwesimintsim District. Two specific objectives were developed from the overall purpose of the study. Regarding the assessment of the relevance of the TBAs, it was found that most of the participants including the expectant mothers, traditional birth attendants and health centre administrators viewed the practice of TBAs as quite relevant. The findings further showed that in spite of the availability of midwives in health centres and continuous training of pre-service nurses, the TBAs were still playing relevant roles in maternal care. The major reasons for the preference of the TBAs services and home delivery over the midwives in the health centres were cost, historic records, experiences, confidence, convenience and sociocultural and traditions. It was also revealed that some of these TBAs play advisory roles to integrate some of their patients into the formal health system. Other reasons included the mode of delivery, the attitude of midwives, and the ill-treatments of expectant mothers. It is therefore concluded that despite the availability of midwifery education and presence in communities, the practice of traditional birth attendants remain quite relevant. It was further concluded that cost of health, experience, trust, proximity, attitude of midwives and socio-cultural factors were the major reasons why the practice of traditional birth attendants is still relevant.

With reference to the second objective which focused on the challenges of TBAs, this paper found that the main challenges associated with traditional birth attendants practice were logistical inadequacy, difficulty in handling complications and challenges of acceptance by the midwives and pre-service midwives who would eventually come out from colleges to practice. The paper also revealed that some of practices of traditional birth attendants were not hygienic. Some even deliver with their bare hands and plastic bags due to lack of appropriate logistics. Furthermore, although the trained TBAs were ready to sterilise their equipment, logistics to do so were not available. Another major challenge was the difficulty in handling complications they identify on expectant mothers. The TBAs indicated that often receive resistance and bullies from the midwives when the send complicated cases of their patients to the health centers. It was also found that some of the midwives and preservice nurses were not aware of the legalization of the practice of some of the TBAs. Again, it was also evident that some of these TBAs find it difficult to compromise on their traditions. It was concluded that logistical inadequacies, difficulty in handling complications and strained relationship with midwives were the major challenges to the practice of the traditional birth attendants. It was also concluded that resistance and bullies from the midwives make integrating the practice of traditional birth attendants into the main health system difficult.

\subsection{Recommendations}

Given the findings of this paper coupled with the conclusions drawn, it is recommended that policy makers should review and strengthen the partnership program and co-ordination between the traditional birth attendants and the midwives in the main the health system so as to build health relationship. This would make it easier for these TBAs to make referrals and some cases accompany their patients to health centers. The ministry of health (MOH) and its partners is advised to resource the TBAs in order that they can observe hygiene in their practice. Doing so would not only benefit the mothers and the babies but also the TBAs themselves. Policy makers are also advised to project a long term goal that would give birth to a midwifery program that would allow the midwives to be integrated as part of the communities they serve. This may be in a form of assisting in home delivery rather than taking the expectant mothers to the health centers. Eventually, the system may weed the TBAs practice completely. 
It is suggested that policy makers should institute policies to check mistreatment of expectant mothers and traditional birth attendants in the various health centers. Appropriate sanctions should be part of this policy to minimise the inhumane treatment revealed in this study. There should be a partnership with the various midwifery training colleges to create awareness of the TBAs program. This would defray the extreme negative perceptions about the TBAs. It is further recommended that management and administrators of midwifery training institutions should liaise with the ministry of health to model their program or design seminars to educate the pre-service midwives of new development in their practice including the TBAs program. The awareness would likely influence their attitude. Management of midwifery training institutions should widen the scope of the pre-service midwives practical to include visit to some of the trained TBAs. This would create friendly atmosphere between them, learn the practice and traditions of these TBAs and design strategies to communicate change to these TBAs tactfully.

\subsection{References}

Abbaszadeh, A., Borhani, F., \& Asadi, N. (2011). Effects of health belief model-based video training about risk factors on knowledge and attitude of myocardial infarction patients after discharge. Journal of Research in Medical Sciences, 16(2).

Adatara, P., Afaya, A., Baku, E. A., Salia, S. M., \& Asempah, A. (2018). Perspective of Traditional Birth Attendants on Their Experiences and Roles in Maternal Health Care in Rural Areas of Northern Ghana. International journal of reproductive medicine, 2018.

Asweto, C. O., Aluoch, J. R., Obonyo, C. O., \& Ouma, J. O. (2014). Maternal Autonomy, Distance to Health Care Facility and ANC Attendance Findings from Madiany Division of Siaya County, Kenya.

Ayele, K., Tesfa, B., Abebe, L., Tilahun, T., \& Girma, E. (2012).Self care behavior among patients with diabetes in Harari, Eastern Ethiopia: the health belief model perspective. PloS one, 7(4), e35515.

Crissman, H. P., Engmann, C. E., Adanu, R. M., Nimako, D., Crespo, K., \& Moyer, C. A.(2013). Shifting norms: pregnant women's perspectives on skilled birth attendance and facility-based delivery in rural Ghana. African journal of reproductive health, 17(1), 15-26.

Ebuehi, O. M., \& Akintujoye, I. A. (2012). Perception and utilization of traditional birth attendants by pregnant women attending primary health care clinics in a rural Local Government Area in Ogun State, Nigeria. Int $\mathbf{J}$ Womens Health, 4, 25-34

Darko, M. L. (2015). To Practice or Not to Practice: Perspectives of Traditional Birth Attendants and Local Communities in The Ga-West Municipality on the New Reproductive and Child Health (Rch) Policy in Ghana (Doctoral dissertation, University of Ghana).

Dzakpasu, S., Soremekun, S., Manu, A., ten Asbroek, G., Tawiah, C., Hurt, L., ... \& Kirkwood, B. R. (2012). Impact of free delivery care on health facility delivery and insurance coverage in Ghana's Brong Ahafo Region. PloS one, 7(11), e49430.

Ganle, J. K., Parker, M., Fitzpatrick, R., \& Otupiri, E. (2014). A qualitative study of health system barriers to accessibility and utilization of maternal and newborn healthcare services in Ghana after user-fee abolition. BMC pregnancy and childbirth, 14(1), 1.

Green, E. C., \& Murphy, E. (2014). Health belief model. The Wiley Blackwell Encyclopedia of Health, Illness, Behavior, and Society. Sage Publications Ltd, London, UK

Kaingu, C. K., Oduma, J. A., \& Kanui, T. I. (2011). Practices of traditional birth attendants in Machakos District, Kenya. Journal of Ethnopharmacology, 137(1), 495-502.

Kumbani, L., Bjune, G., Chirwa, E., \& Odland, J. Ø. (2013). Why some women fail to give birth at health facilities: a qualitative study of women's perceptions of perinatal care from rural Southern Malawi. Reproductive health, $10(1), 9$.

Moyer, C. A., Adongo, P. B., Aborigo, R. A., Hodgson, A., Engmann, C. M., \& DeVries, R. (2014). "It's up to the woman's people": how social factors influence facility-based delivery in Rural Northern Ghana. Maternal and child health journal, 18(1), 109-119.

Oshonwoh, F. E., Nwakwuo, G. C., \& Ekiyor, C. P. (2014). Traditional birth attendants and women's health practices: A case study of Patani in Southern Nigeria. Journal of Public Health and Epidemiology, 6(8), 252261.

Pfeiffer, C., \& Mwaipopo, R. (2013). Delivering at home or in a health facility? health-seeking behaviour of women and the role of traditional birth attendants in Tanzania. BMC pregnancy and childbirth, 13(1), 1.

Sarmento, D. R. (2014). Traditional birth attendance (TBA) in a health system: what are the roles, benefits and challenges: a case study of incorporated TBA in Timor-Leste. Asia Pacific family medicine, 13(1), 1.

Shah, N. M., Brieger, W. R., \& Peters, D. H. (2010). Can interventions improve health services from informal private providers in low and middle-income countries? A comprehensive review of the literature. Health Policy and Planning, 26(4), 275-287.

Titaley, C. R., Dibley, M. J., \& Roberts, C. L. (2012). Type of delivery attendant, place of delivery and risk of early neonatal mortality: analyses of the 1994-2007 Indonesia Demographic and Health Surveys. Health 
Policy and Planning, 27(5), 405-416.

Tones, K., Robinson, Y. K., \& Tilford, S. (2013). Health education: effectiveness and efficiency. Springer.

Vyagusa, D. B., Mubyazi, G. M., \& Masatu, M. (2013). Involving traditional birth attendants in emergency obstetric care in Tanzania: policy implications of a study of their knowledge and practices in Kigoma Rural District. International journal for equity in health, 12(1), 83. 